\title{
Comparison of Two Measurement Devices for Obtaining Horizontal Force-Velocity Profile Variables During Sprint Running
}

Erin Harper Feser ${ }^{1,2}$, Kyle Lindley ${ }^{3}$, Kenneth Clark $^{4}$, Neil Bezodis ${ }^{5}$, Christian Korfist ${ }^{1}$, and John Cronin ${ }^{1}$

\author{
${ }^{1}$ Sports Performance Research Institute New Zealand (SPRINZ) at AUT Millennium, Auckland University of \\ Technology, New Zealand \\ ${ }^{2}$ College of Health Solutions, Arizona State University, USA \\ ${ }^{3}$ School of Biological and Health Systems Engineering, Arizona State University, USA \\ ${ }^{4}$ Department of Kinesiology, West Chester University, USA \\ ${ }^{5}$ A-STEM Research Centre, Swansea University, UK \\ Correspondence: Erin H Feser, Sports Performance Research Institute New Zealand (SPRINZ), AUT Millennium, \\ Auckland University of Technology, Private Bag 92006, Auckland 1142, New Zealand. E-mail: \\ erinfeser@gmail.com
}




\begin{abstract}
This study established the magnitude of systematic bias and random error of horizontal force-velocity (Fv) profile variables obtained from a 1080 Sprint compared to that obtained from a Stalker ATS II radar device. Twenty high-school athletes from an American football training group completed a $30 \mathrm{~m}$ sprint while the two devices simultaneously measured velocity-time data. The velocity-time data were modelled by an exponential equation fitting process and then used to calculate individual F-v profiles and related variables (theoretical maximum velocity, theoretical maximum horizontal force, slope of the linear F-v profile, peak power, time constant tau, and horizontal maximal velocity). The devices were compared by determining the systematic bias and the $95 \%$ limits of agreement (random error) for all variables, both of which were expressed as percentages of the mean radar value. All bias values were within $6.32 \%$, with the 1080 Sprint reporting higher values for tau, horizontal maximal velocity, and theoretical maximum velocity. Random error was lowest for velocity-based variables but exceeded $7 \%$ for all others, with slope of the F-v profile being greatest at $\pm 12.3 \%$. These results provide practitioners with the information necessary to determine if the agreement between the devices and the magnitude of random error is acceptable within the context of their specific application.
\end{abstract}

\title{
KEYWORDS
}

Force-velocity profiling, bias, random error, sprinting, acceleration 


\section{INTRODUCTION}

Sprint running is an important athletic ability for field-based sport play, athletics events, and even some winter sports (e.g. bobsledding). When working to improve speed during athlete training, coaches and sport scientists can use technology to monitor training volume ${ }^{1}$, provide feedback ${ }^{13}$, regulate training session loading parameters ${ }^{9}$, gather performance insights ${ }^{18}$, and record mechanical capabilities ${ }^{8,16}$. However, coaches and sport scientists should understand the accuracy and limitations of the devices they choose to use. In doing so, they can better understand the diagnostic value of the technology and determine how different devices can be used interchangeably to provide comparable information.

Spatio-temporal measurement of athlete sprint running performance is frequently used by coaches and sport scientists used to determine the horizontal force-velocity (F-v) relationship during sprint running. 19,20,22 A Stalker ATS radar device (Applied Concepts, Dallas, TX, USA), which can be used to record velocity-time data during sprint running at $46.875 \mathrm{~Hz}$, is often the technology of choice to calculate the

horizontal F-v relationship ${ }^{24}$ and has been used in research with sprint ${ }^{18}$, rugby ${ }^{7,14}$, and soccer ${ }^{22}$ athletes. The intraday reliability ${ }^{5,22}$ and validity of the technology to measure velocity ${ }^{5}$ and split times ${ }^{10,17}$ have been previously confirmed.

A 1080 Sprint device (1080 Motion, Lidingö, Sweden) is another piece of technology that has become available for commercial use and can measure an athlete's velocity-time curve during sprint running. The 1080 Sprint features a servo motor attached to a spooled cable that can be used with the accompanying software as a robotic resistance or towing device. The system uses an optical encoder that is attached to the motor axis to measure the speed of the cable movement which is recorded by the accompanying software at a sampling rate of $333 \mathrm{~Hz}$. It is assumed that the speed of cable can be used as a surrogate measure for athlete velocity during linear sprint running since the cable is attached to the athlete with a waist harness. The manufacturer reports the velocity and distance measurement accuracy to be $\pm 0.5 \%$ and $\pm 5 \mathrm{~mm}$, respectively. ${ }^{2}$ However, these measurements were taken under controlled laboratory conditions which differs from how the cable moves during use with athletes. The measurement accuracy 
of the 1080 Sprint compared to a radar device (Stalker Pro II) has been assessed during $30 \mathrm{~m}$ sprints with highly trained sprinters. ${ }^{25}$ However, minimal information was reported regarding the methodological approach, statistical analysis, and findings. The coefficients of variation were reported as $5.11 \%$ for $0-10$ $\mathrm{m}$ time, $4.32 \%$ for $10-20 \mathrm{~m}$ time, and $1.51 \%$ for $20-30 \mathrm{~m}$ time but no information was provided regarding the between-device measurement bias and variation values, the device measurement mean and standard deviation values, or other derived metrics such as those used in force-velocity profiling. ${ }^{25}$ Thus, minimal information is currently available regarding the measurement accuracy of the 1080 Sprint, particularly for use in force-velocity profiling.

Considering the recent research interest in the 1080 Sprint as a training tool ${ }^{6,11,12,15,21,25}$ and measurement device ${ }^{9}$ and given its increasing use by coaches and sport scientists, it seems prudent to establish the agreement between data calculated from the 1080 Sprint and an alternative technology already widely used in practice, a radar device. More specifically, it is of interest to understand if measures collected with one device can be compared to measures collected by the other device in situations where athletes may train in facilities which have different devices or in a facility which has both devices. Similarly, it is important to understand how to evaluate data reported in research from the different devices. The findings of this study would provide researchers, coaches, and sport scientists with the necessary information to determine if the agreement between the devices is acceptable within the context of their specific application and, thus, potentially allow for interchangeable use between devices. Therefore, the purpose of this study was to establish the magnitude of systematic bias and random error of horizontal F-v profile variables obtained from 1080 Sprint to those obtained from a widely used radar device (i.e. Stalker ATS II) during a $30 \mathrm{~m}$ sprint run.

\section{MATERIALS AND METHODS}

Twenty athletes volunteered to participate in this study (mean \pm standard deviation; age: $16.5 \pm 0.51$ years, stature: $180 \pm 6.73 \mathrm{~cm}$, body mass: $71.2 \pm 8.11 \mathrm{~kg}$ ). The athletes were all members of the same high-school American football training group. Inclusion criteria required athletes to be currently training 
and categorised as a position player other than an offensive or defensive lineman. Athletes were excluded if they were under the age of 16 , had a current or previous injury that may be further aggravated by participating in the study, or did not pass the Physical Activity Readiness Questionnaire. All study procedures were approved by the host University Institutional Review Board. Participants provided written informed consent and parental assent before participating in the study.

\section{Procedures}

The testing session started with a warm-up protocol that mimicked the athletes' usual practice session preparation, which included time for a self-directed warm-up routine and coach-directed running drills. Following the warm-up, each athlete completed two maximal effort $30 \mathrm{~m}$ sprints. Given the aims of the study, the second trial was used for analysis so that only one trial from any given individual was included in the dataset, and the first trial was stored in the event a technological glitch or operator error rendered the second trial unusable. The sprints were performed from a two-point, split stance position. Athletes were instructed to remain still prior to initiating the forward movement of the sprint start and were allowed to go when they felt ready. Two devices were simultaneously used to measure athlete velocity, a Stalker ATS II radar device and a 1080 Sprint motorised resistance device. The radar device (Stalker ATS II, Applied Concepts, Dallas, TX, USA) was positioned $5 \mathrm{~m}$ directly behind the starting line at a height of $1 \mathrm{~m}$ to approximate the athletes' centre of mass location. STATS software (Version 5.0.2.1, Stalker ATS II, Applied Concepts, Dallas, TX, USA) was used to collect the radar data at $46.875 \mathrm{~Hz}$. The cable of the 1080 Sprint was attached to the athlete with a waist harness and the device was placed on the floor $1.5 \mathrm{~m}$ behind the starting line. A diagram of the experimental set-up is provided as Figure 1. Per manufacturer recommendations, all trials were completed with the 1080 Sprint in Isotonic mode. The minimum load setting of $1 \mathrm{~kg}$ was utilised, necessary for the device to function and thought to be required to maintain tension on the cable. Quantum software (1080 Motion, Lidingö, Sweden) was used to collect the 1080 Sprint data at $333 \mathrm{~Hz}$. 
The raw velocity-time data was exported from both software programmes. A custom-made MATLAB script (MATLAB R2019b, The MathWorks, Inc., Natick, Massachusetts, USA) was used to calculate individual linear F-v profiles by first fitting the velocity-time data up to the estimated $30 \mathrm{~m}$ end-of-sprint with an exponential equation following the method presented in Morin et al. ${ }^{20}$ This included the use of the time shift correction which ignored all velocity data lower than $1.5 \mathrm{~m} / \mathrm{s}$ and extended the model fit backwards to estimate the instant of movement onset for all sprint trials. Following, outlier samples were identified by a residual function to remove all data points $\geq \pm 2 \times$ standard deviation of the residual (i.e. approximately $5 \%$ of the data points) and the final exponential equation was fit to these data after a single iteration of outlier removal. From this final exponential fit, time constant tau and horizontal maximal velocity $\left(\mathrm{V}_{\mathrm{Hmax}}\right)$ were obtained, and then the variables of theoretical maximum velocity $\left(\mathrm{V}_{0}\right)$; theoretical maximum horizontal force $\left(\mathrm{F}_{0}\right)$, peak power $\left(\mathrm{P}_{\mathrm{max}}\right)$, and slope of the $\mathrm{F}-\mathrm{v}$ profile $\left(\mathrm{S}_{\mathrm{Fv}}\right.$; expressed relatively to body mass) were computed following the method previously proposed and validated. ${ }^{20,22}$

[Figure 1 here]

\section{Statistical Analysis}

Means and standard deviations were calculated to represent the centrality and spread of the calculated variables. The variables calculated from the 1080 Sprint velocity-time data were compared to the variables calculated from the radar by determining the bias (mean measurement difference between the devices, 1080 Sprint - Radar $)$ and random error $(1.96 \times$ standard deviation of the differences between the devices), and the $95 \%$ limits of agreement. ${ }^{4}$ To standardise the comparison of the bias and random error values between the different variables of interest, the bias and random error values were also expressed as a percentage of the mean radar value, thus, setting the radar values as reference for comparison purposes. Considering that the random error calculation only represents the maximum random error likely observed in $95 \%$ of cases, the corresponding typical error of estimate was also calculated as standard deviation of the differences between the devices and expressed as a percentage of the mean radar value. Further, graphical representation with Bland-Altman plots were used to show the measurement difference between 
the devices for each sample with the $95 \%$ limits of agreement against the mean of the two measurement devices. To provide a visual standardisation between the different variables for the Bland-Altman plots, both bounds of the y-axis were set to whichever of $\pm 15 \%$ of the mean radar measure had a greater absolute magnitude.

\section{RESULTS}

The velocity-time data from the 1080 Sprint was unable to be modelled by the exponential function within the known time domain of the recorded sprint for five athletes. This was identified by the modelled velocity starting to increase from $0 \mathrm{~m} / \mathrm{s}$ at a time instant which occurred clearly before there had been any movement in the raw data, i.e. the exponential model identified movement onset to occur before any movement had occurred based on the raw data signal. Figure 2 provides an example of one of these athletes' sprint trial where the exponential modelled velocity (dashed line) starts to increase from $0 \mathrm{~m} / \mathrm{s}$ before a time which was known to be prior to any movement based on the raw 1080 Sprint data. These trials were therefore removed from all analyses resulting in 15 remaining athletes. The exponential modelling of the velocity-time data was fitted with an average $R^{2}=0.97$ and all $R^{2}>0.96$ for the radarderived data and an average $R^{2}=0.97$ and all $R^{2}>0.91$ for the 1080 Sprint-derived data.

Mean and standard deviation of the variables calculated are presented in Table 1 along with the bias and random error values. The Bland-Altman plots are displayed in Figure 3. A positive bias, indicating a higher measurement by the 1080 Sprint, was found for tau, $\mathrm{V}_{\mathrm{Hmax}}$, and $\mathrm{V}_{0}$. For $\mathrm{P}_{\max }$, the bias measurement resulted in a value of $0.00 \mathrm{~W} / \mathrm{kg}$, however, the corresponding random error value $( \pm 0.94 \mathrm{~W} / \mathrm{kg})$ represented a range of $\pm 7.09 \%$ of the mean $\mathrm{P}_{\max }$ value measured by the radar. Further, the random error was largest for tau, $\mathrm{F}_{0}$, and $\mathrm{S}_{\mathrm{Fv}}$ representing $\pm 9.98 \%, \pm 9.41 \%$, and $\pm 12.3 \%$ (respectively) of the mean value measures by the radar. These random error values corresponded to a typical error of estimate of $5.09 \%, 4.80 \%$, and $6.30 \%$ for the tau, $\mathrm{F}_{0}$, and $\mathrm{S}_{\mathrm{Fv}}$ measures, respectively. For $\mathrm{V}_{\mathrm{Hmax}}, \mathrm{V}_{0}$, and $\mathrm{P}_{\max }$, the typical error of estimate values were $1.29 \%, 1.49 \%$, and $3.62 \%$, respectively. The velocity-time data 
simultaneously recorded for one athlete's sprint trial along with the corresponding exponential model can be found in Figure 4.

[Figure 2 here]

[Table 1 here]

[Figure 3 here]

[Figure 4 here]

\section{DISCUSSION}

This study established the magnitude of systematic bias and random error between horizontal F-v profile variables obtained from a 1080 Sprint to that obtained from a radar during a $30 \mathrm{~m}$ sprint run. The calculation of systematic bias for the horizontal F-v profile variables resulted in positive bias (indicating a higher measurement by the 1080 Sprint than the radar device) for tau, $\mathrm{V}_{\mathrm{Hmax}}$, and $\mathrm{V}_{0}$. All bias values were within a $6.32 \%$ difference between the devices. The largest relative magnitudes of random error measured between the two devices were found for tau $( \pm 9.98 \%)$ and $\mathrm{S}_{\mathrm{Fv}}( \pm 12.3 \%)$.

While some lack of agreement is generally expected when quantifying performance measures from different measurement methods, careful consideration of the amount by which the methods differ is important for practical interpretation. ${ }^{4}$ When a systematic bias is present, practitioners can utilise the bias value as a standardised offset between the measurement methods. For example, in this study, the 1080 Sprint produced lower mean $\mathrm{F}_{0}$ values resulting in a bias of $-0.14 \mathrm{~N} / \mathrm{kg}$. Moving forward, practitioners could systematically increase the $\mathrm{F}_{0}$ values measured by the 1080 Sprint by $0.14 \mathrm{~N} / \mathrm{kg}$ to correspond to the value that would be recorded by the radar for this athlete population. However, practitioners should not simply proceed with this approach without considering the magnitude of random error present to give insight into the range in which most differences between the two devices will lie. For example, for the $\mathrm{P}_{\max }$ results in this study, although a systematic bias of $<0.01 \mathrm{~W} / \mathrm{kg}$ was identified, the random error value 
$\left( \pm 0.94 \mathrm{~W} / \mathrm{kg}\right.$ ) represented a range of $\pm 7.09 \%$ of the mean $\mathrm{P}_{\max }$ radar value. Therefore, although the devices would on average yield comparable Pmax values across a number of trials, any two trials could be over $14 \%$ different if they occurred at the opposing limits of the random error, and practitioners should be are of this in the context of their specific application.

The horizontal F-v relationship is useful for gaining insight into the sprinter's mechanical effectiveness during sprint running but is reliant on a macroscopic model of the centre of mass velocity. ${ }^{22}$ In this study, $25 \%$ of the sprint trials captured by the 1080 Sprint device were unable to be modelled with the exponential equation fitting process. Simple visual inspection (post-analysis) of the raw velocity data recorded by the 1080 Sprint appears to show-an unusually rapid increase in velocity very early in the trial (see Figure 2 as an example) for some athletes. It is possible that the velocity data recorded by the 1080 Sprint for some athletes does not well match their true centre of mass velocity during this initial portion of the sprint, and this likely led to the onset of the sprint being modelled to occur at an unrealistic instant for five of the athletes in this current study owing to the simple nature of the model fitting process. Given the nature of the 1080 Sprint, this may have been exacerbated by any preparatory movement of the torso prior to the final forward initiation of the sprint, particularly given the relatively inexperienced nature of the athletes tested. A similar limitation in accurately tracking centre of mass motion during the initial steps has been previously reported with velocity estimates from a laser-based device ${ }^{3}$, and this would theoretically also be the case for the radar, but neither are reliant on the attachment of, and tension within, a cable. On the 1080 Sprint machine a cable load setting is required for machine operation. In this study, the lowest load setting $(1 \mathrm{~kg})$ was used to reduce restriction of the athletes' maximal performance and in an attempt to record true maximal performance values. However, with this load setting, slack is easily developed in the line and this could be an additional contributing factor to any error between the measured velocity values and the true velocity of the athlete's movement. A greater load setting would increase the tension between the athlete and the machine to reduce excessive motion of the cable. But it is unknown how a higher load setting could hamper maximal performance resulting in a different F-v 
profile calculation and limiting the comparison to measures from different devices. Finally, whilst the additional $1 \mathrm{~kg}$ load was not accounted for in the data processing undertaken in this study and this may have resulted in a slight underestimation of horizontal force production, this would have been consistent across both of the compared devices due to the simultaneous nature of data collection with both devices. However, researchers should account for this in future studies if comparing 1080 Sprint measures to those obtained from other measurement methods (e.g. gold standard force plate measures) and when sprinting unloaded.

The accuracy of the F-v profiling computation is dependent on successful identification of movement onset. ${ }^{23}$ It is important to note that in this study we systematically removed questionable data around movement onset by use of a velocity-based threshold and the model fit was extended backwards to identify movement onset for all sprint trials. This permitted a consistent application of the modelling approach for both the radar and 1080 Sprint data. Thus, the findings of this study are applicable when a similar approach is utilised. Any perceived limitations of the 1080 Sprint presented here should not be viewed as an overarching reflection of the viability of the machine use in general. Rather, this information is a cautionary note of the potential use and application of the data recorded and processed under the conditions described here (i.e. testing of non-sprint specialist, developmental athletes, some of whose two-point start techniques may have led to difficulties in the measurement of accurate initial velocity data from the 1080 Sprint device). Future research should endeavour to identify other processing approaches for F-v profiling with the 1080 Sprint velocity data that are successful for all trials, as well as ensuring that the start techniques of athletes are carefully monitored and controlled.

\section{IMPLICATIONS}

The data presented in this study provide practitioners with the level of systematic bias and measurement error present when using the above-described approach to F-v profiling with velocity data recorded from a 1080 Sprint and Stalker ATS II radar. Individual decisions should be made on whether the potential for error is acceptable within the intended application, including the variables of interest and the specific 
within- or between-athlete comparisons being made. For example, a practitioner may deem it acceptable

to use the two devices interchangeably to provide feedback during a training session but choose to utilise

one device consistently for periodic performance testing.

\section{REFERENCES}

1. Aughey RJ. Applications of GPS technologies to field sports. Int J Sports Physiol Perform. 2011;6:295-310.

2. Bergkvist C, Svensson M, Eriksrud O. Accuracy and repeatability of force, position and speed measurement of 1080 Quantum and 1080 Sprint. https://1080motion.com/science/summary-ofspeed-force-accuracy-1080-sprint-1080-quantum-2015/: 1080 Motion;2015.

3. Bezodis NE, Salo AIT, Trewartha G. Measurement error in estimates of sprint velocity from a laser displacement measurement device. Int J Sports Med. 2012;33:439-444.

4. Bland JM, Altman DG. Measuring agreement in method comparison studies. Stat Methods Med Res. 1999;8:135-160.

5. Chelly SM, Denis C. Leg power and hopping stiffness: relationship with sprint running performance. Medicine \& Science in Sports \& Exercise. 2001;33(2):326-333.

6. Clark K, Cahill M, Korfist C, Whitacre T. Acute kinematic effects of sprinting with motorized assistance. J Strength Cond Res. 2019.

7. Cross MR, Brughelli M, Brown SR, et al. Mechanical properties of sprinting in elite rugby union and rugby league. Int J Sports Physiol Perform. 2015;10:695-702.

8. Cross MR, Brughelli M, Samozino P, Morin J-B. Methods of power-force-velocity profiling during sprint running: A narrative review. Sports Med. 2017;47:1255-1269.

9. Cross MR, Lahti J, Brown SR, et al. Training at maximal power in resisted sprinting: Optimal load determination methodology and pilot results in team sport athletes. PLOS ONE. 2018;13(4):e0195477.

10. di Prampero PE, Fusi S, Sepulcri L, Morin JB, Belli A, Antonutto G. Sprint running: a new energetic approach. The Journal of Experimental Biology. 2005;208:2809-2816.

11. Helland C, Haugen T, Rakovic E, et al. Force-velocity profiling of sprint athletes: single-run vs. multiple-run methods. Eur J Appl Physiol. 2019;119:465-473.

12. Lahti J, Jiménez-Reyes P, Cross MR, et al. Individual sprint force-velocity profile adaptations to in-season assisted and resisted velocity-based training in professional rugby. Sports 2020;8(5).

13. Liebermann DG, Katz L, Hughes MD, Bartlett RM, McClements J, Franks IM. Advances in the application of information technology to sport performance. Journal of Sport Sciences. 2002;20(10):755-769.

14. Macadam P, Simperingham K, Cronin J. Acute kinematic and kinetic adaptations to wearable resistance during sprint acceleration. J Strength Cond Res. 2017;21(5):1297-1304.

15. Mangine GT, Huet K, Williamson C, et al. A resisted sprint improves rate of force development during a 20-m sprint in athletes. J Strength Cond Res. 2018;32(6):1531-1537.

16. Mendiguchia J, Edouard P, Samozino P, et al. Field monitoring of sprint power-force-velocity profile before, during and after hamstring injury: two case reports. J Sports Sci. 2016;34(6):535541.

17. Morin J-B, Jeannin T, Chevallier B, Belli A. Spring-mass model characteristics during sprint running: Correlation with performance and fatigue-induced changes. Int J Sports Med. 2006;27:158-165.

18. Morin J-B, Bourdin M, Edouard P, Peyrot N, Samozino P, Lacour J. Mechanical determinants of 100-m sprint running performance. Med Sci Sports Exerc. 2012;43(9):1680-1688. 
19. Morin J-B, Samozino P. Interpreting power-force-velocity profiles for individualized and specific training. Int J Sports Physiol Perform. 2016;11:267-272.

20. Morin J-B, Samozino P, Murata M, Cross M, Nagahara R. A simple method for computing sprint acceleration kinetics from running velocity data: Replication study with improved design. $J$ Biomech. 2019;94:82-87.

21. Rakovic E, Paulsen G, Helland C, Eriksrud O, Huagen T. The effect of individualised sprint training in elite female team sport athletes: A pilot study. Journal of Sport Sciences. 2018;36(24):2802-2808.

22. Samozino P, Rabita G, Dorel S, et al. A simple method for measuring power, force, velocity properties, and mechanical effectiveness in sprint running. Scand J Med Sci Sports. 2016;26:648658.

23. Samozino P. Biomechanics of training and testing: Innovative concepts and simple field methods. Cham, Switzerland: Springer International Publishing AG; 2018.

24. Simperingham KD, Cronin JB, Ross A. Advances in sprint acceleration profiling for field-based team-sport athletes: Utility, reliability, validity and limitations. Sports Med. 2016;46:1619-1645.

25. Thompson KMA, Whinton AK, Ferth S, Spriet LL, Burr JF. Moderate load resisted sprints do not improve subsequent sprint performance in varsity level sprinters. J Strength Cond Res.

2021;35(1):72-77. 


\begin{tabular}{cc|c} 
Radar & 1080 Sprint & \multicolumn{1}{c}{ Start Line } \\
\hline & & \\
Sprint Direction $>$ & \\
\hline
\end{tabular}

Figure 1. A diagram of the experimental set-up. 


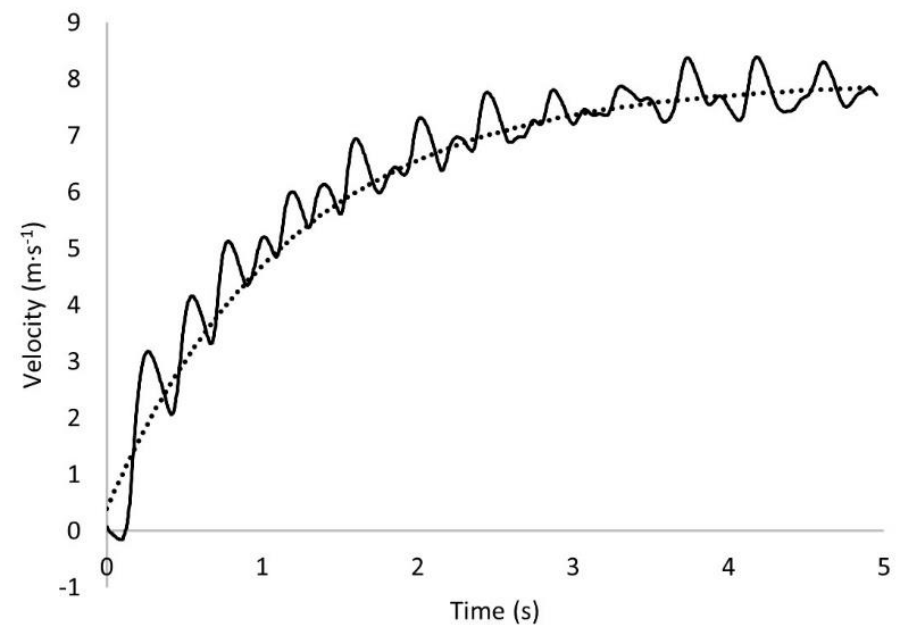

Figure 2. An example of the horizontal velocity recorded by the 1080 Sprint (solid line) and the corresponding exponential modelled velocity (dashed line) which could not be modelled within the time domain of the recorded sprint for one athlete's sprint trial. 

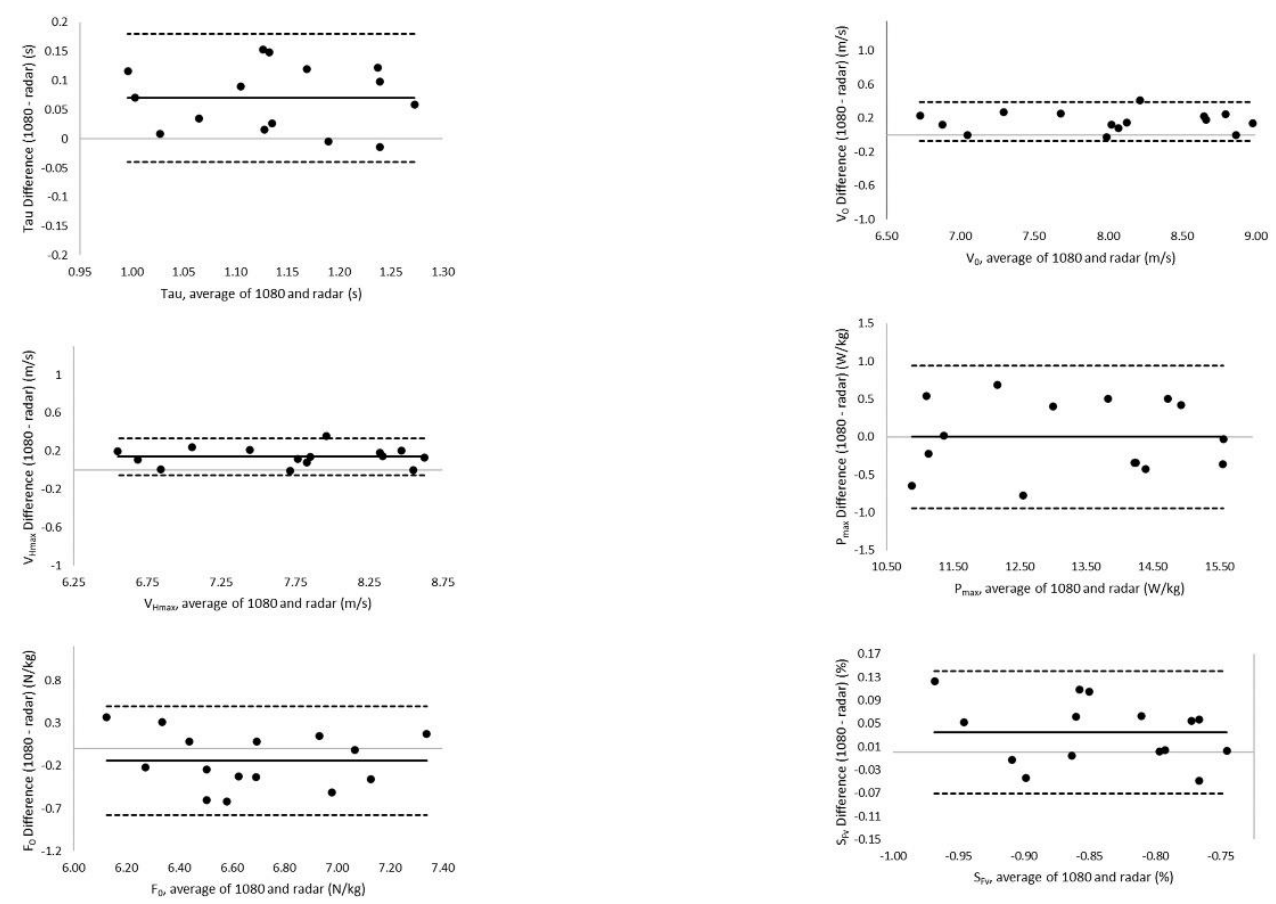

Figure 3. Bland-Altman plots representing the bias and random error for each measurement variable. 


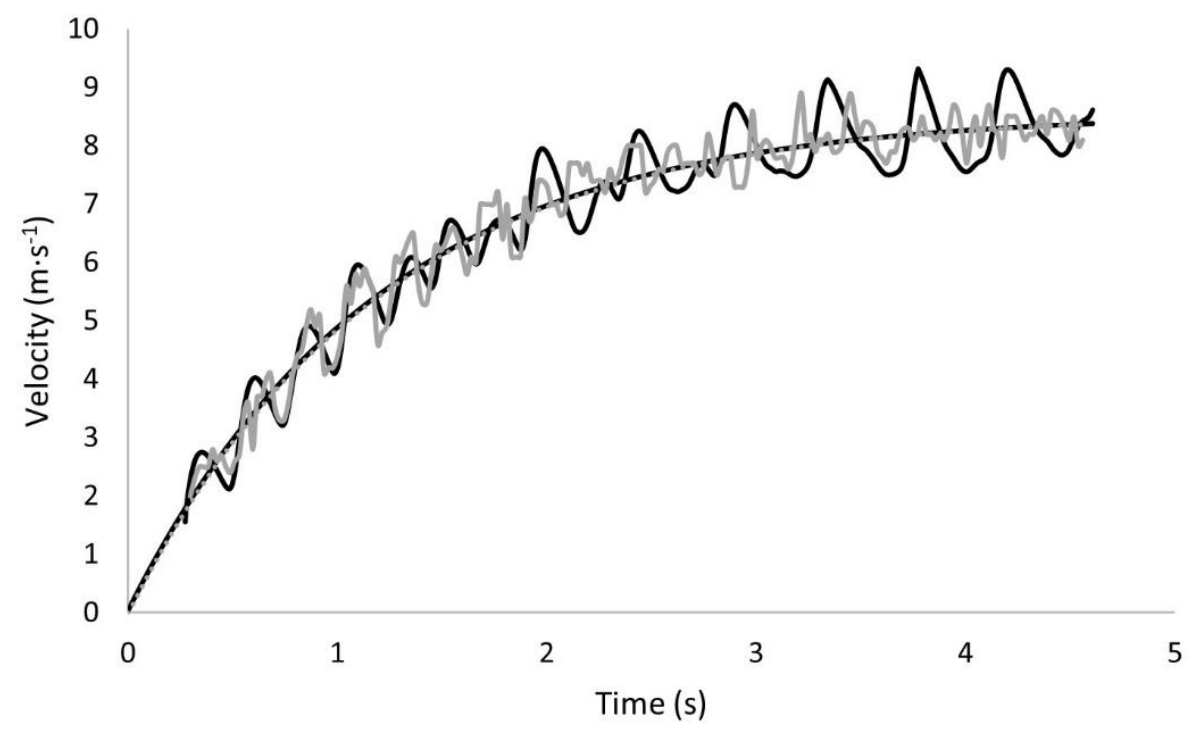

Figure 4. The horizontal velocity simultaneously recorded by the radar (grey) and 1080 Sprint (black) for one athlete's sprint trial is presented with the corresponding exponential modelled velocity. 
Table 1. Device measurements (mean \pm SD) and between device bias, random error, and limits of agreement.

\begin{tabular}{lccccccc}
\hline & 1080 Sprint & Radar & Bias* & Bias \% & $\begin{array}{c}\text { Random } \\
\text { Error }\end{array}$ & $\begin{array}{c}\text { Random } \\
\text { Error \% }\end{array}$ & 95\% LOA \\
\hline Tau $(\mathbf{s})$ & $1.17 \pm 0.09$ & $1.10 \pm 0.09$ & 0.07 & $6.32 \%$ & \pm 0.11 & $\pm 9.98 \%$ & $(-0.04,0.18)$ \\
$\mathbf{V}_{\text {Hmax }}\left(\mathbf{m} \cdot \mathbf{s}^{-\mathbf{1}}\right)$ & $7.80 \pm 0.69$ & $7.66 \pm 0.69$ & 0.14 & $1.85 \%$ & \pm 0.19 & $\pm 2.54 \%$ & $(-0.05,0.34)$ \\
$\mathbf{F}_{\mathbf{0}}(\mathbf{N} / \mathbf{k g})$ & $6.61 \pm 0.36$ & $6.75 \pm 0.40$ & -0.14 & $-2.05 \%$ & \pm 0.64 & $\pm 9.41 \%$ & $(-0.77,0.50)$ \\
$\mathbf{V}_{\mathbf{0}}\left(\mathbf{m} \cdot \mathbf{s}^{-1}\right)$ & $8.08 \pm 0.74$ & $7.92 \pm 0.74$ & 0.16 & $2.02 \%$ & \pm 0.23 & $\pm 2.92 \%$ & $(-0.07,0.39)$ \\
$\mathbf{S}_{\mathbf{F v}}(\boldsymbol{\%})$ & $-0.82 \pm 0.07$ & $-0.86 \pm 0.08$ & 0.04 & $-4.08 \%$ & \pm 0.11 & $\pm 12.3 \%$ & $(-0.07,0.14)$ \\
$\mathbf{P}_{\max }(\mathbf{W} / \mathbf{k g})$ & $13.3 \pm 1.69$ & $13.3 \pm 1.69$ & $<0.01$ & $0.00 \%$ & \pm 0.94 & $\pm 7.09 \%$ & $(-0.94,0.94)$ \\
\hline
\end{tabular}

*A positive bias indicates a higher measurement by the 1080 Sprint device than the radar device; LOA = limits of agreement. The bias $\%$ and random error $\%$ are calculated as a percentage of the mean radar value. 